\title{
Novel strategies for targeting innate immune responses to influenza
}

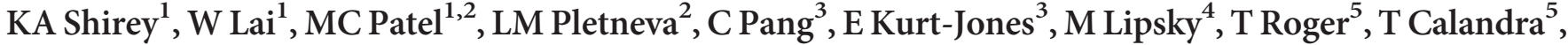 \\ KJ Tracey $^{6}$, Y Al-Abed ${ }^{7}$, AG Bowie $^{8}$, A Fasano $^{9}$, CA Dinarello ${ }^{10}$, F Gusovsky ${ }^{11}$, JCG Blanco ${ }^{2}$ and SN Vogel $^{1}$
}

We previously reported that TLR4 ${ }^{-1-}$ mice are refractory to mouse-adapted A/PR/8/34 (PR8) influenza-induced lethality and that therapeutic administration of the TLR4 antagonist Eritoran blocked PR8-induced lethality and acute lung injury (ALI) when given starting 2 days post infection. Herein we extend these findings: anti-TLR4- or-TLR2-specific IgG therapy also conferred significant protection of wild-type (WT) mice from lethal PR8 infection. If treatment is initiated $3 \mathrm{~h}$ before PR8 infection and continued daily for 4 days, Eritoran failed to protect WT and TLR $4^{-/-}$mice, implying that Eritoran must block a virus-induced, non-TLR4 signal that is required for protection. Mechanistically, we determined that (i) Eritoran blocks high-mobility group B1 (HMGB1)-mediated, TLR4-dependent signaling in vitro and circulating HMGB1 in vivo, and an HMGB1 inhibitor protects against PR8; (ii) Eritoran inhibits pulmonary lung edema associated with ALI; (iii) interleukin (IL)-1 $\beta$ contributes significantly to PR8-induced lethality, as evidenced by partial protection by IL-1 receptor antagonist (IL-1Ra) therapy. Synergistic protection against PR8-induced lethality was achieved when Eritoran and the antiviral drug oseltamivir were administered starting 4 days post infection. Eritoran treatment does not prevent development of an adaptive immune response to subsequent PR8 challenge. Overall, our data support the potential of a host-targeted therapeutic approach to influenza infection.

\section{INTRODUCTION}

Influenza continues to evolve with new antigenic variants emerging annually, as exemplified by the last several influenza seasons in which the recommended vaccine was considerably less efficacious than predicted. ${ }^{1-4}$ Therefore, there remains a pressing need to develop alternatives to the annual influenza vaccines and antiviral agents currently used to mitigate the effects of influenza infection. Multiple pattern recognition receptors, including TLR3, TLR4, TLR7, TLR8, and TLR10, and the intra-cytosolic sensor RIG-I have been implicated in influenza-induced disease, although TLR10 is not functional in mice. ${ }^{5-12} \mathrm{CD} 14$ is required for influenza-induced cytokine production by mouse macrophages, independent of TLR2 and TLR4. ${ }^{13}$ In addition, influenza-infected MyD88 ${ }^{-1-}$ and MyD88/TRIF doubly deficient mice show a marked reduction of pulmonary cytokine production when compared with WT mice, ${ }^{5,11,12}$ indicating the important role of these TLR signaling pathways in disease.

Imai et al. ${ }^{14}$ proposed that chemical or microbial insults trigger NADPH-dependent reactive oxygen species that generate a host-derived oxidized phospholipid, oxidized 1-palmitoyl-2-arachidonyl-phosphatidylcholine (OxPAPC), in lungs. They concluded that regardless of the initial sensing involved in pathogen recognition, OxPAPC initiates a common TLR4-, TRIF-, and IL-6-dependent pathway in macrophages that leads to acute lung injury (ALI). We showed that treatment of influenza-infected mice with Eritoran, the most potent, synthetic lipid A analog known, ${ }^{15}$ blocked influenza-induced lethality and ALI. When administered daily to WT mice for 5 days, starting on days 2, 4, or 6 post infection, Eritoran

\footnotetext{
${ }^{1}$ Department of Microbiology and Immunology, University of Maryland, Baltimore, Maryland, USA. ${ }^{2}$ Sigmovir Biosystems, Rockville, Maryland, USA. ${ }^{3}$ Department of Medicine, University of Massachusetts Medical School, Worcester, Massachusetts, USA. ${ }^{4}$ Pathology Research, University of Maryland, Baltimore, Maryland, USA. ${ }^{5}$ Infectious Diseases Service, Centre Hospitalier Universitaire Vaudois and University of Lausanne, Lausanne, Switzerland. ${ }^{6}$ Department Biomedical Science, The Feinstein Institute for Medical Research, Manhasset, New York, USA. ${ }^{7}$ Department of Medicinal Chemistry, The Feinstein Institute for Medical Research, Manhasset, New York, USA. ${ }^{8}$ School of Biochemistry and Immunology, Trinity Biomedical Sciences Institute, Trinity College Dublin, Dublin 2, Ireland. ${ }^{9}$ Mucosal Immunology and Biology Research Center, MGH for Children, Boston, Massachusetts, USA. ${ }^{10}$ Division of Infectious Diseases, University of Colorado Denver, Aurora, Colorado, USA and ${ }^{11}$ Eisai, Andover, Massachusetts, USA. Correspondence: SN Vogel (svogel@som.umaryland.edu)
} 
treatment significantly improved survival and clinical symptoms, while decreasing ALI, OxPAPC accumulation, the cytokine storm, and systemic inflammation. ${ }^{16}$ Herein we extend our previous findings by characterizing the role of TLR4 and TLR2 in influenza-induced lethality and ALI, and provide new insights into the molecular mechanisms underlying protection by modulators of these important TLRmediated inflammatory pathways.

\section{RESULTS}

\section{Eritoran in influenza models}

We previously reported that TLR $4^{-1-}$ mice were refractory to mouse-adapted influenza PR8, ${ }^{16,17}$ and have now confirmed that TLR4 ${ }^{-1-}$ mice are also refractory to a more pathogenic, mouse-adapted pandemic H1N1 strain, ma.Ca/04 (ref. 18; Supplementary Figure 1A online). We also showed previously that therapeutic treatment of PR8-infected, WT mice with Eritoran significantly protected against lethality, and attenuated ALI, findings now reproduced in C57BL/6 mice infected with the ma.Ca/04 strain (Supplementary Figure 1B,C). Eritoran also protected PR8-infected BALB/c mice (data not shown). We found that an additional daily dose of Eritoran (i.e., administered once vs. twice daily), starting 4 days post infection, failed to improve the protection achieved with a single dose daily (data not shown). Together, these data expand our previous observations that therapeutic treatment of mice with Eritoran protects against lethality with additional influenza strains and newly explored conditions of infection. The following experiments were designed to provide mechanistic insights into Eritoran-mediated protection and to identify the pathways that contribute to influenza-induced disease that are affected by Eritoran treatment.

\section{Elucidation of signaling requirements underlying influenza-induced lethality and protection by Eritoran}

Eritoran blocks TLR4 signaling by binding in the deep hydrophobic pocket of its co-receptor MD2, thereby blocking ligand-induced dimerization. ${ }^{19}$ To validate the role of TLR4 in PR8-induced lethality, mice were treated with a highly specific anti-TLR4 antibody ${ }^{20}$ (Figure 1a). Anti-TLR4 IgG, but not an isotype-matched control IgG, administered intravenously (i.v.) on day 2 only or on days 2 and 4 post PR8 infection, protected mice against lethal infection (Figure 1b) and elicited significantly improved clinical scores $(P<0.004$; Figure 1c). This result confirms that TLR4 signaling is, indeed, central to influenza-induced lethality and clinical symptoms.

TLR4 activates both the MyD88- and TRIF-dependent signaling pathways. ${ }^{8}$ One of the central conclusions of Imai et al. ${ }^{14}$ was that TLR4-mediated ALI induced by inactivated H5N1 influenza or the host-derived oxidized phospholipid OxPAPC is entirely TRIF-dependent. However, MyD88 has been implicated in the host response to influenza. ${ }^{9,12}$ IRAK4, the first enzyme recruited to MyD88, initiates signaling leading to $I K K \alpha / \beta / \gamma$ complex activation, IKB $\alpha$ phosphorylation, and ultimately, nuclear factor- $\kappa \mathrm{B}$ activation. The TRIF pathway drives IRF3 activation and results in delayed nuclear factor- $\kappa \mathrm{B}$
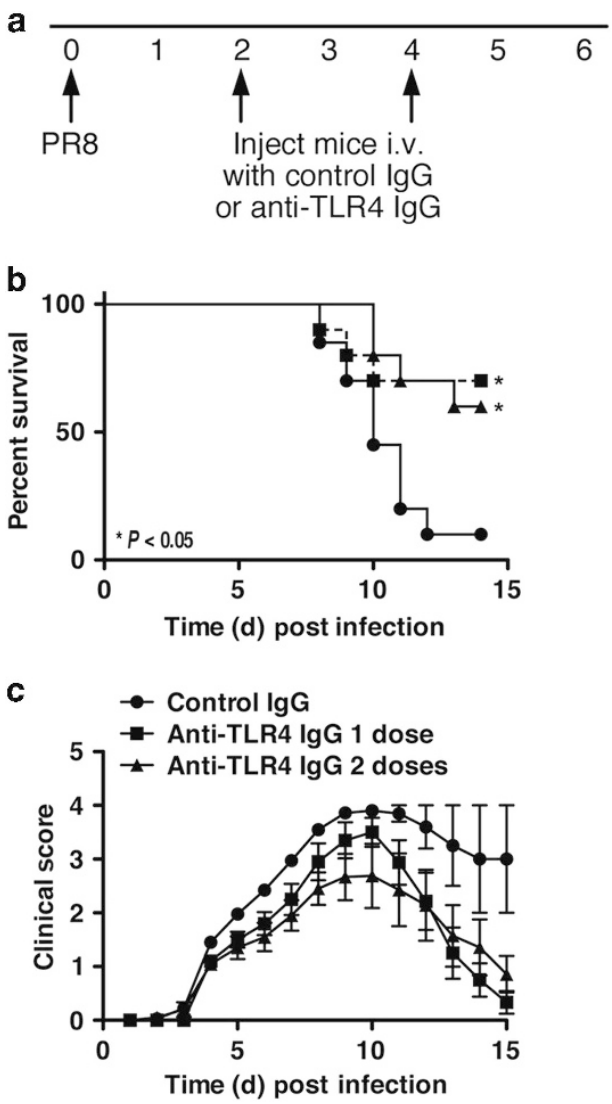

Figure 1 Anti-TLR4 IgG treatment protects mice from lethal influenza challenge. (a) C57BL/6J mice were infected with mouse-adapted influenza strain PR8 ( 7,500 TCID ${ }_{50}$, i.n.). Mice received either control IgG or a highly specific anti-TLR4 IgG (2 mg/mouse; i.v.) once (day 2 only) or twice (days 2 and 4). Survival (b) and clinical scores (c) were monitored daily. Each graph represents the combined results of two separate experiments (5 mice/treatment group/experiment). i.n., intranasally; i.v., intravenously; TCID, tissue culture infective dose.

activation, independent of IRAK4. ${ }^{21}$ To delineate the downstream pathway(s) underlying the host response to influenza and the protective mechanisms of Eritoran, we compared PR8induced lethality and the efficacy of Eritoran in IRAK4 kinase dead knock-in (IRAK4 ${ }^{\mathrm{KDKI}}$ ) mice that have a catalytically inactive form of IRAK4 that blocks MyD88-dependent signaling, vs. TRIF $^{-1-}$ mice. IRAK4 ${ }^{\mathrm{KDKI}}$ mice exhibited a slightly delayed mean time to death compared with WT mice, and Eritoran therapy resulted in $\sim 60 \%$ survival compared with $\sim 90 \%$ in WT mice (Figure 2a). Interestingly, $\mathrm{TRIF}^{-1-}$ mice were more resistant to PR8 infection than WT or IRAK4 ${ }^{\mathrm{KDKI}}$ mice $\left(\sim 50 \%\right.$ survival), but not as refractory as TLR $4^{-1-}$ mice. ${ }^{16,17}$ However, treatment with Eritoran significantly improved the survival of $\mathrm{TRIF}^{-1-}$ mice to WT levels $(P<0.001$; Figure $2 \mathbf{b})$. VIPER is a TLR4-inhibitory peptide derived from the A46 protein of vaccinia virus that has been shown to inhibit both MyD88- and TRIF-dependent TLR4 signaling by binding to and targeting the sorting adaptors TIRAP and TRAM. ${ }^{22}$ When WT mice were infected with PR8 and treated therapeutically with either a cell-permeating VIPER peptide 9R-VIPER, or Eritoran, 9R-VIPER treatment resulted 

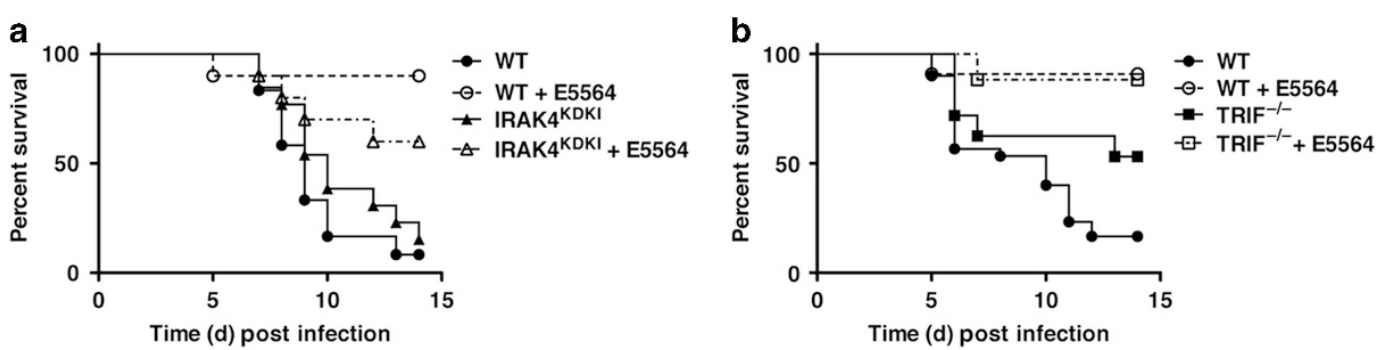

Figure 2 Effect of Eritoran on IRAK4 ${ }^{\mathrm{KDKI}}$ and TRIF ${ }^{-1-}$ mice. Wild-type (WT) C57BL/6J (a and b), IRAK4 ${ }^{\mathrm{KDKI}}$ (a) and TRIF ${ }^{-1-}$ (b) mice were infected with mouse-adapted influenza strain PR8 ( 7,500 TCID 5 , i.n.). Mice received vehicle (saline; i.v.) or Eritoran (E5564; $200 \mu \mathrm{g} / \mathrm{mouse}$; i.v) daily from day 2 to day 6 post infection. Survival was monitored for 14 days. Data shown are combined results of two to three separate experiments (5-10 mice/treatment group/experiment). i.n., intranasally; i.v., intravenously; TCID, tissue culture infective dose.

in partial protection $(\sim 50 \%)$, consistent with a role for TIRAP and/or TRAM in protection (Supplementary Figure 2). Thus, altogether, both MyD88- and TRIF-dependent pathways contribute to influenza-mediated disease and Eritoraninduced protection.

We reported previously that TLR2 ${ }^{-1-}$ mice were similarly sensitive to WT mice for PR8-induced lethality. However, unlike WT mice, Eritoran therapy failed to protect TLR2 ${ }^{-1-}$ mice; thus, TLR2 was presumed to be a direct or indirect target for Eritoran. ${ }^{16}$ To confirm the role of TLR2 in influenzainduced disease, we used a monoclonal antibody directed against TLR2 (clone T2.5) that blocks TLR2-mediated signaling in vivo. ${ }^{23}$ Groups of mice were either treated with anti-TLR2 or with an isotype control antibody $3 \mathrm{~h}$ prior and 1 day post PR8 infection, while two other groups of mice received antiTLR2 or control antibody on days 2 and 4 post PR8 infection (Figure 3a). Similar to the protection achieved with anti-TLR4 IgG (Figure 1), treatment of PR8-infected WT mice with antiTLR2 antibody significantly protected against lethality when administered on days 2 and 4 post infection $(P<0.001$; Figure 3b); however, anti-TLR2 treatment was not effective when administered earlier. These results suggest the presence of a TLR2 agonist released late after PR8 infection contributes to lethality.

To extend these findings, WT, TLR2 ${ }^{-1-}$, TLR $^{-1-}$, and TLR2/4 double-knockout mice were infected with a sublethal dose $\left(\mathrm{LD}_{10}\right)$ of PR8 and monitored for 14 days. The TLR2/4 double-knockout mice were much more susceptible than the WT or individual knockout mice (Supplementary Figure 3A). ALI was significantly worse in TLR2/4 double-knockout mice than in WT, with inflammatory infiltrates throughout the parenchyma and alveolar spaces (composed of neutrophils and lymphocytes) (Supplementary Figure 3B). These findings suggest that a TLR2 agonist induced early during virus infection is necessary for the resistance of TLR4 ${ }^{-1-}$ mice to lethal PR8 infection.

\section{Timing of Eritoran treatment is critical for protection}

Neither differential influenza replication (Figure 4a, left panel) nor the levels of inducible interferon (IFN)- $\beta$ mRNA (Figure 4a, right panel) accounted for the resistance of the a Therapeutic regimen
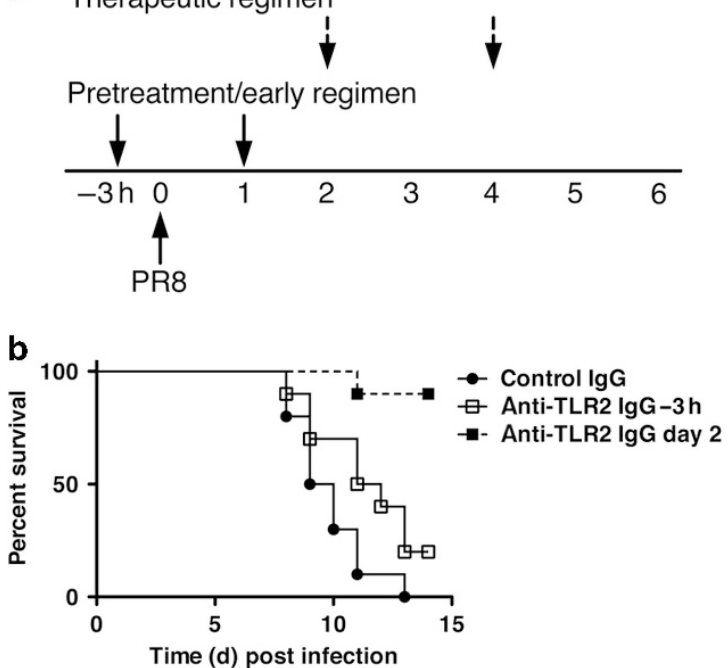

Figure 3 Anti-TLR2 IgG treatment protects mice from lethal influenza challenge. (a) Experimental protocol. C57BL/6J mice were either treated with isotype control IgG or with anti-TLR2 (T2.5; $100 \mu \mathrm{g} / \mathrm{ms}$; i.v.) $3 \mathrm{~h}$ before and 1 day post infection, or on days 2 and 4 post infection. Survival (b) was monitored daily. Data shown are combined results of two separate experiments (5 mice/treatment group/experiment). i.v., intravenously.

TLR4 ${ }^{-1-}$ mice to PR8 infection. Eritoran therapy protected PR8-infected WT mice (Figure $\mathbf{4 b}, \mathbf{c}$, open circle, left panel), but did not affect the resistance of TLR4 ${ }^{-1-}$ mice (Figure $\mathbf{4 b}$,d; open circle, right panel), as we reported previously. ${ }^{16}$ However, when Eritoran treatment was initiated prophylactically $(3 \mathrm{~h}$ before PR8 infection) and continued daily for an additional 4 days (Figure $\mathbf{4 b}$, pretreatment/early regimen; closed squares), WT mice were not protected from lethality (Figure $\mathbf{4 c}$, closed square, left panel). This finding implies that an early influenzainducible, but late-acting mediator of lethality and ALI must be the target of Eritoran in WT mice. Surprisingly, this identical regimen rendered TLR4 ${ }^{-1-}$ mice susceptible to PR8 infection (Figure 4b,d, closed square, right panel), indicating that a nonTLR4 target of Eritoran is necessary for the resistance of TLR4 ${ }^{-1-}$ mice to PR8 infection, consistent with our data obtained with the TLR2/4 double-knockout mice. Pre-/early 

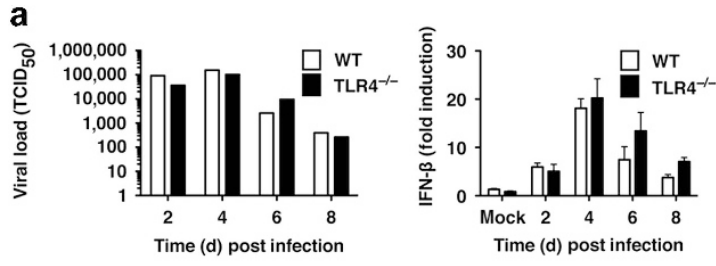

b
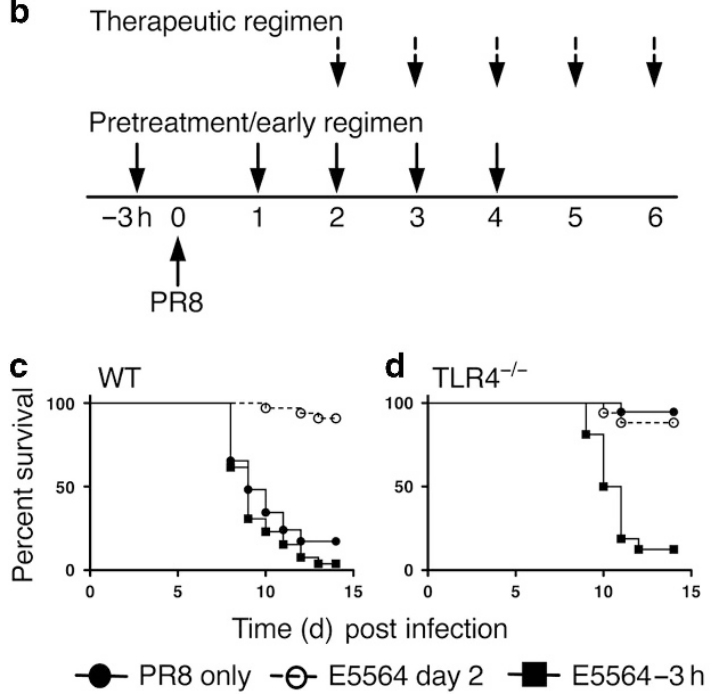

Figure 4 Pretreatment of wild-type (WT) or TLR4 ${ }^{-1-}$ mice with Eritoran does not protect from lethal influenza challenge. (a) WT and TLR4 ${ }^{-1-}$ mice were infected with mouse-adapted influenza strain PR8 $(\sim 7,500$ $\mathrm{TCID}_{50}$, i.n.) and killed on days 2, 4, 6, and 8 post infection. Lungs were collected and assayed for viral titers and interferon (IFN)- $\beta$ mRNA. Data shown are combined results of two separate assays (4-5 mice/group/ experiment). (b) Basic experimental protocol used to compare pretreatment/early vs. therapeutic treatment with Eritoran in PR8-infected mice. C57BL/6J WT and TLR4 ${ }^{-1-}$ mice were either untreated (solid circles) or treated with Eritoran starting $3 \mathrm{~h}$ before infection and on day 1 for 4 successive days ( $3 \mathrm{~h}$; solid squares; pretreatment/early regimen), or starting 2 days after infection for 5 successive days (open circles; therapeutic regimen). (c and d) Mice were monitored daily for survival. The data represent the combined results of two separate experiments (5-6 mice/treatment/experiment). i.n., intranasally; TCID, tissue culture infective dose.

treatment with Eritoran had no effect on the susceptibility of $\mathrm{CD} 14^{-1-}$ or $\mathrm{TLR}^{-1-}$ mice (data not shown).

P5779, an HMGB1 antagonist, blocks influenza-mediated lethality

We previously reported that lungs of Eritoran-treated, PR8infected mice (as shown in Figure 1a) showed blunted cytokine induction as well as accumulation of OxPAPC, ${ }^{16}$ a dangerassociated molecular pattern (DAMP) shown by Imai et al. ${ }^{\mathrm{I}}$ to mediate ALI by its action on macrophages through TLR4. HMGB1, a DAMP first implicated in endotoxicity and Gram-negative sepsis, ${ }^{24}$ has been reported to be released during severe influenza infection ${ }^{25}$ and to activate TLR4 by binding to the TLR4 co-receptor MD2. ${ }^{26}$ HMGB1-stimulated WT murine macrophages induced MyD88- (tumor necrosis factor- $\alpha$ ) and TRIF (IFN- $\beta$ )-dependent gene expression that was inhibited by Eritoran in vitro (Figure 5a). Mice infected with PR8 (data not shown) and cotton rats infected with a non-adapted human influenza pdH1N1 strain, exhibited increased circulating HMGB1 that was inhibited by Eritoran treatment in vivo (Figure 5b). Thus, HMGB1, like OxPAPC, may represent a DAMP that is released relatively late after infection that contributes to influenza-induced ALI through TLR4 activation. P5779 is a small-molecule inhibitor of HMGB1 that was shown recently to prevent MD-2/HMGB1 interaction and block HMGB1-induced TLR4 signaling, while not interfering with lipopolysaccharide (LPS)-induced cytokine/ chemokine induction. ${ }^{27}$ P5779 protected mice against hepatic ischemia/reperfusion injury, acetaminophen (APAP) chemical toxicity, and sepsis. ${ }^{27}$ To assess the efficacy of P5779 in influenza infection, WT C57BL/6J mice were infected with PR8 and, 2 days later mice were treated with either Eritoran (E5564), an inactive control peptide, or P5779 for 5 consecutive days. Both Eritoranand P5579-treated mice showed significant survival and lowered clinical scores, while mice treated with the control inhibitor showed higher clinical scores and succumbed to infection (Figure 5c,d).

\section{A PAR 2 antagonist blocks influenza-induced lethality and lung leak}

A host-derived protein, originally termed zonulin (now known to be pre-haptoglobin 2), ${ }^{28}$ was found to increase intestinal permeability by phosphorylation of tight junction proteins. ${ }^{29}$ Signaling was dependent upon protease-activated receptor 2 $\left(\mathrm{PAR}_{2}\right){ }^{28}$ a signaling protein that we have shown previously to interact physically and functionally with TLR4. ${ }^{30} \mathrm{~A}$ zonulin analog peptide antagonist AT-1001 (larazotide acetate) is well tolerated in humans and attenuates gut inflammation associated with celiac disease. ${ }^{31}$ Recently, AT-1001 was also reported to attenuate ALI in mice, induced by intrapulmonary deposition of IgG immune complexes or LPS, by inhibiting phosphorylation of the tight junction protein ZO-1, reducing the number of leukocytes and myeloperoxidase activity in bronchoalveolar lavage fluid. ${ }^{32}$ As both $\mathrm{PAR}_{2}^{-1-}$ and TLR4 ${ }^{-1-}$ mice are comparably refractory to lethal PR8 infection, ${ }^{17}$ we compared the efficacy of AT-1001 vs. Eritoran therapy during a lethal influenza challenge. WT mice were infected with PR8 and treated with vehicle (saline), Eritoran, or AT-1001 for 5 consecutive days starting on day 2 post infection. Treatment of mice with AT-1001 resulted in significant protection and lowered clinical scores, comparably to Eritoran treatment (Figure 6a,b). As AT-1001 reduced the pulmonary edema associated with LPS- or immune complex-induced ALI, ${ }^{32}$ we tested whether Eritoran and AT1001 mediated a decrease in lung edema caused by PR8 infection as measured by wet-to-dry weight ratio. Mice infected with PR8 and treated with vehicle showed significantly higher wet-to-dry ratios than mice infected with PR8 and treated with either Eritoran or AT-1001 (Figure 6c), suggesting that an additional protective effect of Eritoran during influenza infection is to attenuate ALI by blunting pulmonary edema.

\section{Contribution of IL-1 to influenza-induced lethality}

We previously showed that IL-1 $\beta$ mRNA is strongly inhibited in the lungs of PR8-infected, Eritoran-treated mice. ${ }^{16}$ Subsequently, Teijero et al. ${ }^{12}$ reported that influenza-infected 
a
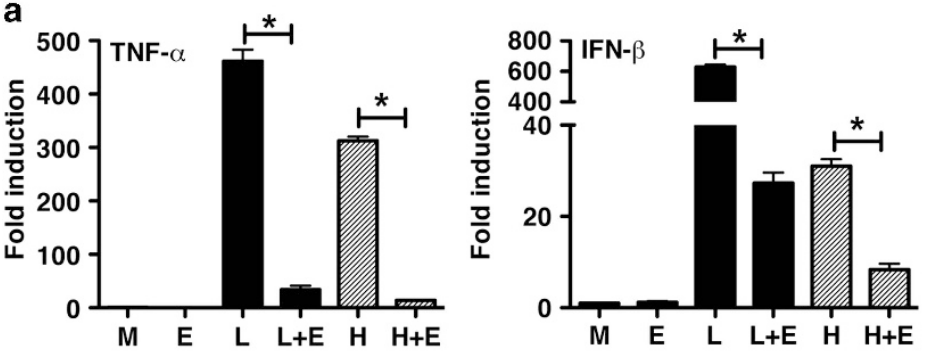

b

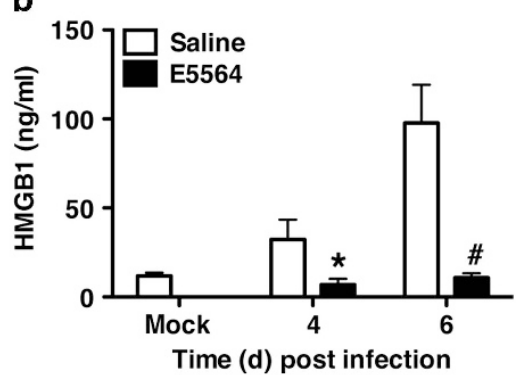

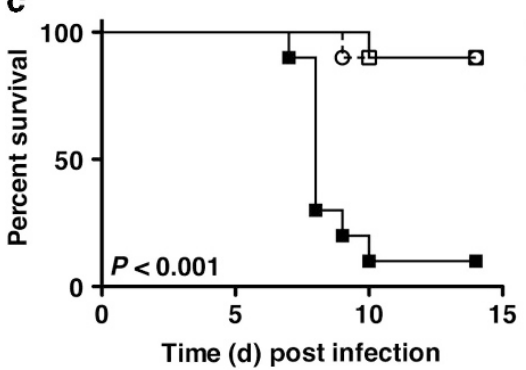

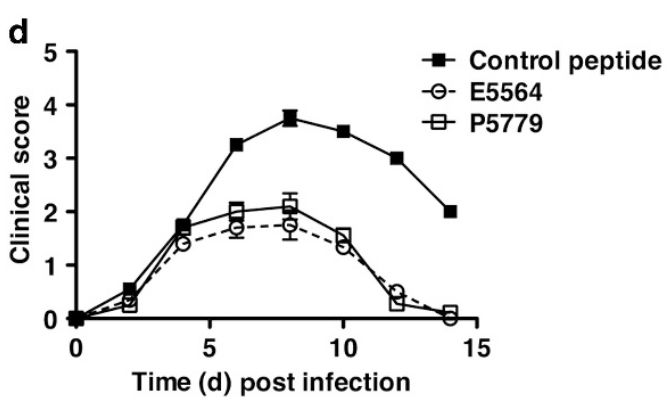

Figure 5 Eritoran blunts HMGB1-induced TLR4 signaling in vitro and influenza-induced HMGB1 release and lethality in vivo. (a) Thioglycollate-elicited C57BL/6J macrophages were treated with medium alone (M) or Eritoran (E; $10 \mathrm{ng} / \mathrm{ml})$ for $1 \mathrm{~h}$, and then with LPS (L; $10 \mathrm{ng} / \mathrm{ml})$ or HMGB1 (H; $1 \mu \mathrm{g} / \mathrm{ml})$ for $2 \mathrm{~h}$. Total RNA was processed and subjected to quantitative real time-PCR for detection of tumor necrosis factor (TNF)- $\alpha$ or interferon (IFN)- $\beta$ mRNA expression. (b) Cotton rats (6-8/time point/treatment) were infected with pdH1N1 A/California/04/09 $\left(9 \times 10^{5} \mathrm{TCID}_{50}\right.$ by i.n.), then treated with Eritoran or saline starting at 2 days post infection. Serum HMGB1 levels were measured by enzyme-linked immunosorbent assay $\left({ }^{*} P<0.05\right)$. (c) C57BL/6J mice were infected with mouse-adapted influenza strain PR8 ( 7,500 TCID 50 , i.n.). Mice received control peptide ( $500 \mu \mathrm{g} / \mathrm{mouse}$; i.p.), Eritoran (E5564; $200 \mu \mathrm{g} / \mathrm{mouse}$; i.v.), or the HMGB1 peptide, P5779 (500 $\mu \mathrm{g} /$ mouse; i.p.) from day 2 to day 6 post infection. Survival (c) and clinical scores (d) were monitored daily for 14 days. Data shown are combined from two experiments (5-10 mice/treatment group/experiment). HMGB1, high-mobility group B1; i.n., intranasally; i.p., intraperitoneally; i.v., intravenously; LPS, lipopolysaccharide; TCID, tissue culture infective dose.
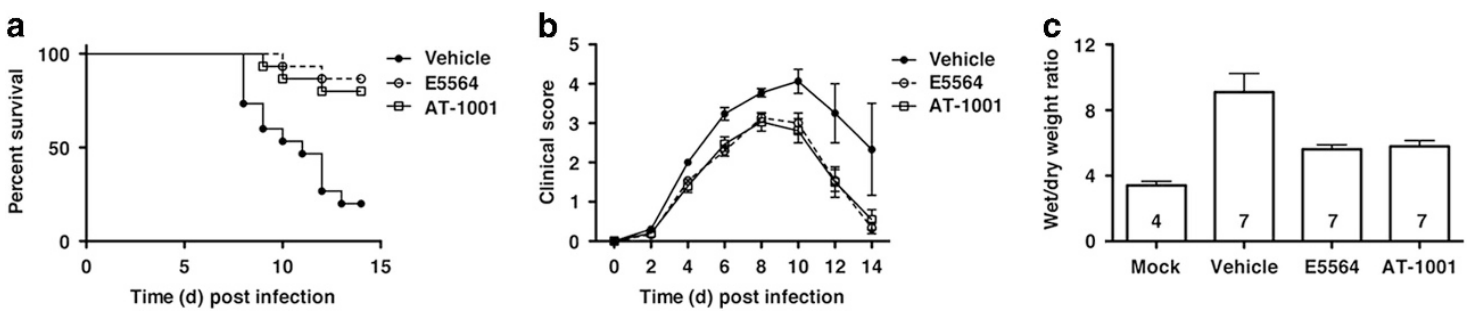

Figure 6 Effect of AT-1001 against lethal influenza challenge. (a) C57BL/6J mice were infected with mouse-adapted influenza strain PR8

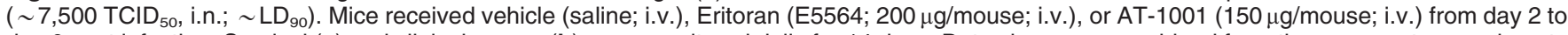
day 6 post infection. Survival (a) and clinical scores (b) were monitored daily for 14 days. Data shown are combined from three separate experiments (5 mice/treatment group/experiment). (c) Lung wet-to-dry (W/D) weight ratio as an index for pulmonary edema after infection. C57BL/6J mice were infected and treated as described above. On day 7 post infection, lungs were collected and lung weights were measured immediately after excision and recorded as wet weight. Lung tissue was air dried for 5-6 days and reweighed until a stable dry weight obtained. The W/D weight ratio was calculated by dividing the wet by dry weight. The $N$ for each group is indicated in each bar. Each vertical bar represents the mean ( \pm s.e.m.). i.n., intranasally; i.v., intravenously; LD, lethal dose; TCID, tissue culture infective dose.

IL-1R $\mathrm{R}^{-/-}$mice exhibited diminished cytokine levels in the bronchoalveolar lavage fluid 2 days post infection (p.i.), but neither ALI nor lethality was evaluated. rIL-1 receptor antagonist (IL-1Ra; generic anakinra) is used clinically to treat highly inflammatory diseases (e.g., rheumatoid arthritis, cryopyrin-associated periodic syndromes, and macrophage activation syndrome). ${ }^{33}$ PR8-infected C57BL/6J WT mice treated therapeutically with $\mathrm{rIL}-1 \mathrm{Ra}$ showed significant, but intermediate, survival (Figure 7a), and clinical scores
(Figure $7 \mathbf{b}$ ), in contrast to the protection afforded by Eritoran. This suggests that while IL-1 $\beta$ participates in mediating influenza-induced disease, other mediators, whose action is inhibited by Eritoran, are likely involved.

\section{Eritoran treatment improves the efficacy of oseltamivir therapy against influenza}

The efficacy of Eritoran administered alone or combined with the approved neuraminidase inhibitor antiviral therapy 

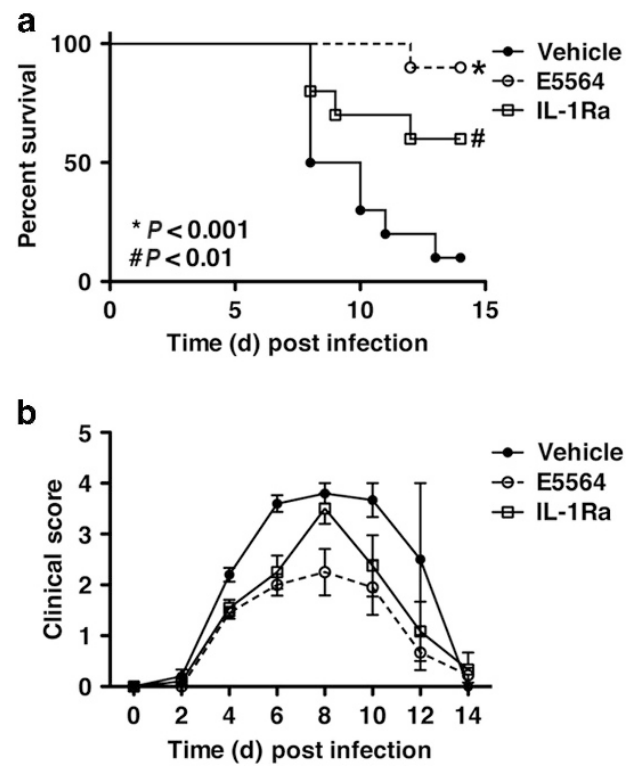

Figure 7 Effect of IL-1Ra against lethal influenza challenge. C57BL/6J mice were infected with mouse-adapted influenza strain PR8 $(\sim 7,500$ $\mathrm{TCID}_{50}$, i.n.). Mice received vehicle (saline; i.v.), Eritoran (E5564; $200 \mu \mathrm{g} /$ mouse; i.v.), or the IL-1Ra (150 $\mu \mathrm{g} / \mathrm{mouse}$; i.v.) from days 2 to 6 post infection. Survival (a) and clinical scores (b) were monitored daily for 14 days. Data shown are combined from two separate experiments (5 mice/ treatment group/experiment). IL-1Ra, interleukin-1 receptor antagonist i.n., intranasally; i.v., intravenously; TCID, tissue culture infective dose.

Tamiflu (oseltamivir) was assessed. To be effective, oseltamivir treatment is recommended within 2 days of the onset of symptoms. ${ }^{34,35}$ As, in our model, mice show clinical symptoms starting at day 3 post infection, ${ }^{16}$ we compared mice treated with vehicle, Eritoran alone, oseltamivir alone, or both Eritoran and oseltamivir starting on days 2, 4, or 6 post infection for 5 consecutive days. When treatment was initiated on day 2 post infection, both agents were highly protective, with no benefit when administered together (Supplementary Figure 4). However, when administration of treatment was delayed until 4 or 6 days post infection, treatment with oseltamivir alone showed little protective effect (particularly when administered starting at day 6), while Eritoran still elicited a significant degree of protection from lethality, as we reported previously. ${ }^{16}$ Importantly, when initiated on day 4, the combined Eritoran-oseltamivir treatment resulted in a significant improvement in survival $(P<0.05$; Supplementary Figure $4 \mathrm{~A})$, as well as significant reduction of viral titers $(P<0.05$; Supplementary Figure 4B). This is consistent with Zheng et al. ${ }^{36}$ who showed that co-administration of celecoxib, a COX-2 inhibitor, and zanamivir improved the survival of influenza-infected mice better than treatment with zanamivir alone. Our previous study showed that Eritoran blunts COX-2 induction during influenza infection. ${ }^{16}$

\section{Survivors of PR8 infection after Eritoran treatment are protected from lethal reinfection}

Importantly, mice that were protected from PR8 by Eritoran survived secondary PR8 challenge 4 weeks after initial infection, without additional Eritoran therapy (Supplementary Figure $\mathbf{5 A}$ ). The half-life of Eritoran is $\sim 5 \mathrm{~h}$ in rodents, ${ }^{37}$ so it would not be expected to be involved in the protective effect seen when survivors were reinfected 28 days after the first PR8 infection. To test this hypothesis, mice were treated with Eritoran for 5 successive days. Five days after the last Eritoran treatment, mice were infected with PR8. A control group was treated with Eritoran starting 2 days post infection for 5 consecutive days (days 2-6). As shown in Supplementary Figure 5B, the lethality seen in the mice given Eritoran early is equivalent to mice that were given vehicle (saline), supporting the idea that the half-life of Eritoran is too short to mediate protection observed upon rechallenge of survivors (Supplementary Figure 5A). To confirm that the Eritoran-treated, PR8-infected surviving mice develop an adaptive memory response, we repeated the assay from Supplementary Figure $\mathbf{5 A}$ and carried out hemagglutination inhibition assays using the sera from mice infected and treated with Eritoran. In mice that were PR8-infected, followed by Eritoran treatment (days 2-6), strong hemagglutination inhibition titers were observed 4 weeks after infection (ranging from 640 to 2,$560 ; n=5$ ). Thus, the anti-inflammatory effect of Eritoran during primary infection does not prevent development of an adaptive immune response against influenza.

\section{DISCUSSION}

Influenza is a major health concern globally. The virus mutates rapidly, leading to antiviral resistance or altered expression of immunogenic epitopes such that extant vaccines are rendered ineffective. On the basis of our previous studies, ${ }^{16}$ we demonstrated that Eritoran (E5564), a well-tolerated, synthetic TLR4 antagonist, ${ }^{38,39}$ represents a novel therapeutic approach to ameliorate influenza-induced ALI by blocking TLRmediated signaling in response to host-derived DAMPs. Herein we delineate further the cellular and molecular underpinnings for both induction of ALI and its abatement by Eritoran therapy.

Our data show that Eritoran, administered after infection, does not alter the refractoriness of TLR4 ${ }^{-1-}$ mice to influenza infection; ${ }^{16}$ both Eritoran and anti-TLR4 antibody therapy protected WT mice, and, Eritoran binds to both CD14 and MD2 in vitro, ${ }^{16}$ both of which are required for TLR4 signaling. ${ }^{19}$ In addition to 9R-VIPER (Supplementary Figure 2), the cell-permeating TLR2 TIR decoy peptide 2R9, which blocks TLR4, 7, and 9 signaling by blocking recruitment of TIRAP to the TLR TIR domain, ${ }^{40}$ protected WT mice from PR8-induced lethality comparably to Eritoran. Together, these findings strongly implicate TLR4 in both influenza-induced disease and as a target for Eritoran-mediated protection. As Eritoran pre/early treatment renders TLR $4^{-1-}$ mice susceptible to PR8 infection, Eritoran must also interact with a non-TLR4 pattern recognition receptor that is required early for induction of resistance. We postulate that Eritoran, administered by the pre/early treatment, binds CD14 and inhibits transfer of specific PAMPs or DAMPs to TLR2, ${ }^{41}$ TLR3, ${ }^{42}$ TLR7, and/or TLR9 (ref. 43) as CD14 has been shown 
to act as a co-receptor for each of these pattern recognition receptors, and as the latter three have been implicated in the host response to influenza ${ }^{5-12}$ and are capable of inducing IFN- $\beta^{21}$. This hypothesis is supported by our observation that WT and TLR $4^{-1-}$ mice, when treated with Eritoran by the early regimen, are as susceptible as IFN- $\beta^{-1-}$ mice to PR8 infection, ${ }^{16}$ and that $\mathrm{CD} 14^{-1-}$ mice could not be protected by Eritoran pre/early or therapeutic regimens, ${ }^{16}$ presumably because they cannot produce cytokines as suggested by Pauligk et al. ${ }^{13}$ Our previous work showed that 5,6-dimethylxanthenone-4-acetic acid (DMXAA), a potent inducer of IFN- $\beta$, protected WT, but not IFN- $\beta^{-1-}$ mice, from PR8-induced lethality when administered $3 \mathrm{~h}$ before and on day 1 post PR8 infection, ${ }^{44}$ again supporting the need for IFN- $\beta$ early in infection for survival.

The role of TLR2 in Eritoran-mediated protection of PR8-infected mice is enigmatic. Despite the fact that we observed that anti-TLR2 monoclonal antibody, but not control IgG, protected WT mice from PR8 when administered therapeutically, the same anti-TLR2 monoclonal antibody, when administered $-3 \mathrm{~h}$ before and 1 day after PR8 infection, provided only minimal protection. This observation strongly suggests that TLR2, like TLR4, also has a damaging role later in infection. OxPAPC ${ }^{45}$ and HMGB1 (ref. 26) have been reported to be both TLR2 and TLR4 agonists that are induced later in infection. These DAMPs may synergize to increase TLR2 expression and/or TLR2-dependent signaling. Our observation that TLR2/4 double-knockout mice are highly susceptible to sublethal PR8 infection may suggest that the absence of both TLR2 and TLR4 would leave MyD88 more available for IL-1 and IL-18 signaling. Alternatively, it is also possible that TLR2 is required to produce something that mediates resistance to PR8 in $\mathrm{TLR}^{-1-}$ mice.

Eritoran blunts both the influenza-induced 'cytokine storm' and the accumulation of OxPAPC, ${ }^{16}$ an oxidized phospholipid TLR4 DAMP. ${ }^{14}$ Mechanistically, these findings have now been extended by showing that Eritoran (i) blocked HMGB1mediated TLR4-dependent signaling in vitro, HMGB1 release into serum in vivo, and protected comparably to P5779, a highly selective HMGB1 inhibitor; and (ii) inhibited pulmonary lung edema equivalently to AT-1001, an inhibitor of zonulininduced pulmonary edema. ${ }^{32}$ Others have shown that influenza induces necroptosis in lung epithelial cells, ${ }^{46,47}$ and that HMGB1 is released from necroptotic cells. ${ }^{48}$ Therefore, it is possible that treatment of mice with Eritoran blocks release of HMGB1 by blocking necroptosis as well as the subsequent signaling through the TLR4/MD2 complex. We previously reported that lung sections from placebo-treated mice showed significant pathology, including epithelial necrosis, and that Eritoran-treated mice had intact lung epithelia compared with the placebo group. ${ }^{16}$

Eritoran acts on cells that express TLR4/MD2. At this time, we do not know whether stromal cells and/or cells of myeloid lineage are involved in the protective effect. Zhang et al. ${ }^{49}$ recently reported that in addition to its profound effects on the lung, influenza infection affects mucosal epithelium in the intestinal tract, consistent with our report of systemic inflammation induced by PR8 infection, ${ }^{16}$ and our results showing that administration of AT-1001 (larazotide acetate), an inhibitor of intestinal and lung leakage, ${ }^{28,31,32}$ reduced PR8induced mortality. Thus, it is possible that influenza-mediated intestinal leakage underlies changes in gut microbiota that are required for the development of adaptive immunity. ${ }^{50}$ In a model of trauma/hemorrhagic shock, Sodhi et al. ${ }^{51}$ reported that ALI was induced in WT mice, but not in mice selectively engineered to lack TLR4 on gut epithelial cells. In this model, ALI in WT mice could be blocked by neutralizing HMGB1 or by treatment with a small-molecule TLR4 inhibitor. ${ }^{51}$ Additional experiments will be required to test the hypothesis that DAMP release by influenza-induced necrosis of lung epithelial cells acts locally to induce ALI, as well systemically to cause gut leak and an altered microbiota. ${ }^{50}$

In addition, the partial protection achieved by treatment of PR8-infected mice supports a role for IL-1 signaling in PR8induced lethality, as suggested by Tejeiro et al.; ${ }^{12}$ however, the data also suggest a role for additional inflammatory mediators in PR8-induced disease as the protection is partial. Nonetheless, the IL- $1 \alpha$ precursor (pro-IL-1 $\alpha$ ) is expressed in the nuclei of most tissues bound to chromatin. ${ }^{52}$ During an acute ischemic event, the loss of oxygenation and increased acidosis triggers release of the pro-IL- $1 \alpha$ into the cytosol and then into the milieu upon cellular necrosis. Pro-IL-1 $\alpha$ binds to the IL-1R1 and is biologically active, ${ }^{53}$ causing the induction of chemokines that mediate neutrophilic infiltration. Thus, the partial protection elicited by IL-1Ra (Figure 7) may be attributable to the blockade of the interaction of pro-IL- $1 \alpha$ with the IL-1R1, in addition to the downregulation of IL-1 $\beta$ mRNA induced in PR8-infected, Eritoran-treated mice.

Our observation that Eritoran-treated mice that survived initial infection and exhibited blunting of the cytokine storm were protected from secondary influenza infection suggests that Eritoran does not prevent the development of an adaptive immune response. During primary influenza infection, antibody levels are detectable by day 10 post infection and persist above baseline for weeks. ${ }^{54}$ We observed that significant levels of anti-hemagglutinin antibodies were induced in mice infected with PR8 followed by Eritoran therapy on days 2-6, even 4 weeks after infection. Thus, Eritoran does not prevent induction of an adaptive immune response to infection. Although such mice remain refractory to secondary PR8 infection 28 days after the primary infection, future studies will be required to determine whether Eritoran treatment alters the enhanced sensitivity to secondary bacterial infections seen in influenza-infected mice.

Overall, our findings underscore the complex nature of microbe-host inflammatory cell interactions that control the host's ability to respond to invading virus. We provide evidence that multiple receptors on innate immune cells are likely involved; however, it is also likely that unknown interactions among these receptors in response to microbial and host ligands significantly affect, both qualitatively and quantitatively, the host response. 


\section{METHODS}

Reagents. Eritoran (E5564) was provided by Eisai (Andover, MA) and prepared as described previously. ${ }^{16}$ Escherichia coli K235 LPS was prepared as previously described. ${ }^{55}$ The anti-TLR2 IgG and isotype control IgG were purchased from Affymetrix (Santa Clara, CA). Recombinant HMGB1 was provided by Kevin Tracey (Feinstein Institute for Medical Research, Manhasset, NY). 9R-VIPER was synthesized by GenScript (Piscataway, NJ). P5779 and control peptide were provided by Yousef Al-Abed (Feinstein Institute for Medical Research). The anti-TLR4 IgG and isotype control IgG were provided by Thierry Roger and Thierry Calandra (Infectious Diseases Service, Centre Hospitalier Universitaire Vaudois and University of Lausanne, Lausanne, Switzerland). AT-1001 was kindly provided by Alessio Fassano (Division of Pediatric Gastroenterology and Nutrition, MGH, Boston, MA). IL-1Ra (anakinra) was obtained from Sweden Orphan BioVitrum (SOBI), Stockholm, Sweden.

Mice and cotton rats. Male and female 6- to 8-week-old, WT C57BL/ $6 \mathrm{~J}$ mice were purchased from the Jackson Laboratory (Bar Harbor, $\mathrm{ME}$ ). All mice with targeted mutations were bred onto or derived directly from a C57BL/6J background. TLR $4^{-1-}$ mice (provided by Shizuo Akira, Osaka, Japan; bred at UMB (Baltimore, MD) and University of Massachusetts Medical School (Worcester, MA), TRIF $^{-1-}$ (bred at University of Massachusetts Medical School). IRAK4 ${ }^{\mathrm{KDKI}}$ (provided by Lilly Research Laboratories, Indianapolis, IN, bred at UMB), TLR $2^{-1-}$ mice (provided by Shizuo Akira; bred at University of Massachusetts Medical School), and TLR2/TLR4 doubleknockout mice (bred at University of Massachusetts Medical School (Worcester, MA). BALB/cByJ mice were purchased from Jackson Laboratories. All mouse strains were housed and bred in specific pathogen-free conditions. Experiments were conducted in accordance with the guidelines set forth by the University of Maryland, Baltimore and the University of Massachusetts Medical School Department of Animal Medicine and approved by each institute's Institutional Animal Care and Use Committees.

Inbred young adult (4-8 weeks old) cotton rats of both male and female (Sigmodon hispidus) were bred at Sigmovir Biosystems (Rockville, MD). All cotton rat experiments were conducted with Institutional Animal Care and Use Committee approval from Sigmovir Biosystems.

Virus. Mouse-adapted H1N1 influenza A/PR/8/34 virus (PR8; ATCC, Manassas, VA) was grown previously described, ${ }^{56}$ and was kindly provided by Dr Donna Farber (Columbia University, New York, NY). Mouse-adapted H1N1 influenza maCa.04 was provided by Daniel Perez (University of Georgia, Athens, GA). Non-adapted human influenza virus strain $\mathrm{pH} 1 \mathrm{~N} 1$ was obtained and grown as previously described. ${ }^{57}$

Virus challenge and treatments. Mice were infected with mouseadapted influenza virus, strains $\mathrm{A} / \mathrm{PR} / 8 / 34$ intranasally (i.n.; PR8; $\sim 7,500$ tissue culture infective dose (TCID) $)_{50}, 25 \mu \mathrm{l} /$ nares) or maCa.04 ( 2,200 $\mathrm{TCID}_{50}$, i.n.). Two days after infection, mice received either vehicle or E5564 i.v. (Eritoran; $200 \mu \mathrm{g} / \mathrm{mouse}$ in $100 \mu \mathrm{l}$; i.v.), anti-TLR4 $\operatorname{IgG}(2 \mathrm{mg} / \mathrm{mouse}$, i.v. $)$ or its isotype control $\mathrm{IgG}$, anti-TLR2 (T2.4 clone; $100 \mu \mathrm{g} /$ mouse, i.v.) or its isotype control IgG, 9R-VIPER ( $200 \mathrm{nmol}$; intraperitoneally), P5779 or its control peptide $(500 \mu \mathrm{g} / \mathrm{mouse}$; intraperitoneally), AT-1001 (150 $\mathrm{g} / \mathrm{mouse}$, i.v.), IL-1Ra $(150 \mu \mathrm{g} /$ mouse, i.v. $)$, or oseltamivir orally ( $1 \mathrm{mg} /$ mouse) daily (days 2-6). In some experiments, groups of mice were treated with Eritoran or oseltamivir starting at day 4 or 6 post infection and treated for 5 successive days. In some experiments, some groups of mice were treated with E5564 $(200 \mu \mathrm{g} /$ mouse, i.v.) $3 \mathrm{~h}$ before infection with PR8 and then treated for 4 consecutive days starting day 1 post infection (days 1-5). In some experiments, groups of mice were treated with anti-TLR2 (T2.5 clone, $100 \mu \mathrm{g} / \mathrm{mouse}$, i.v.) or its control isotype IgG $3 \mathrm{~h}$ before infection with PR 8 and then treated for a second time at day 1 post infection for a total of two treatments. Mice were monitored daily for survival, weight loss, and clinical signs of illness (e.g., lethargy, piloerection, ruffled fur, hunched posture, rapid shallow breathing, and audible crackling) for 14 days. A clinical score ranging from 0 (no symptoms) to 5 (moribund) was ascribed to each mouse daily. ${ }^{16,58}$

Histopathology. Lungs were inflated and perfused and fixed with $4 \%$ paraformaldehyde. Fixed sections $(8 \mu \mathrm{m})$ of paraffin-embedded lungs were stained with hematoxylin and eosin. Slides were randomized, read blindly, and examined for tissue damage, necrosis, apoptosis, and inflammatory cellular infiltration.

TLR2/4 double-knockout mouse studies. C57BL/6J WT, TLR4 ${ }^{-/-}$, TLR2 $^{-1-}$, and TLR2/4 double-knockout mice were $8-12$ weeks of age at the time of infection. Influenza $\mathrm{A} / \mathrm{PR} / 8 / 34$ (Charles River Laboratories, Wilmington, MA) virus stocks were diluted in sterile phosphate-buffered saline and kept on ice before use. Mice were anesthetized with isoflurane and infected 40,000 plaque forming units $(30 \mu l$; i.n.) per mouse. In two assays, mice were monitored for survival for 14 days post infection. In another assay, mice were killed on day 5 post infection and the lungs collected for pathology. Lungs were inflated in situ with $1 \mathrm{ml}$ of formalin (10\% formaldehyde) obtained from the University of Massachusetts Medical School Morphology Core. The inflated lungs were fixed for $24-48 \mathrm{~h}$, after which they were transferred to phosphate-buffered saline. Lungs were bisected vertically and processed for paraffin embedding by the University of Massachusetts Medical School Morphology Core. Slides were prepared and hematoxylin and eosin-stained for histological analysis.

Lung wet-to-dry weight ratio. The lung wet-to-dry weight ratio was used as an index of pulmonary edema after infection with influenza in mice that were untreated or treated with either E5564 or AT-1001. On day 7 post infection, mice were killed and dissected for the total lung, and the lung weight was measured immediately after excision (wet weight). Lung tissue was then air dried for 5-6 days and reweighed every day until a dry weight was stable and this acted as the final dry weight. The wet-to-dry weight ratio was calculated by dividing the wet by the final dry weight. ${ }^{59}$

Quantitative real-time PCR. Total RNA isolation and quantitative real-time PCR were performed as previously described. ${ }^{16}$ Levels of mRNA for specific genes are reported as relative gene expression normalized to mock-infected lungs.

Macrophage cell cultures and treatment. Thioglycollate-elicited peritoneal macrophages from $\mathrm{C} 57 \mathrm{BL} / 6 \mathrm{~J}$ WT mice were enriched as described ${ }^{16}$ after plating in 12 -well $\left(2 \times 10^{6}\right.$ cells/well $)$ tissue culture plates. Macrophages were pretreated with Eritoran $(10 \mathrm{ng} / \mathrm{ml})$ for $1 \mathrm{~h}$ and then stimulated with LPS $(10 \mathrm{ng} / \mathrm{ml})$ or HMGB1 $(1 \mu \mathrm{g} / \mathrm{ml})$ for $2 \mathrm{~h}$.

HMGB1 serum levels. Cotton rats were infected with $9 \times 10^{4} \mathrm{TCID}_{50}$ of pH1N1 i.n. on day 0 . On day 2 post infection, animals were treated with saline (mock) or with $37.33 \mathrm{mg} / \mathrm{kg}$ of Eritoran i.v., daily. Blood samples were collected on days 0,4 , and 6 post infection, and serum was used for measuring HMGB1 levels using an ELISA kit according to the manufacturer's protocol (IBL International, Toronto, Ontario, Canada)

Viral titration. Virus titers were obtained from supernatants of lung homogenates of PR8-infected mice that were either left untreated or treated with Eritoran only, oseltamivir only, or both Eritoran and oseltamivir, and collected on day 7 post infection and expressed at $\mathrm{TCID}_{50} / \mathrm{ml}$ as described previously. ${ }^{4}$

Hemagglutination inhibition assay. Before serological analysis, mice sera were treated with receptor-destroying enzyme (Denka Seiken, Catalog \# 370013, Tokyo, Japan). Serum was mixed with receptordestroying enzyme and incubated at $37^{\circ} \mathrm{C}$ for $20 \mathrm{~h}$, followed by heat inactivate at $56{ }^{\circ} \mathrm{C}$ for $30 \mathrm{~min}$. Finally, $0.6 \mathrm{ml}$ of $1 \times$ phosphatebuffered saline was added to give a 1:10 dilution of the initial sample. To quantify hemagglutination inhibition activity, twofold serial 
dilutions of treated sera in 96-well plates were incubated with four hemagglutinating units of PR8 virus at room temperature for $15 \mathrm{~min}$. Turkey red blood cells at $0.5 \%$ in phosphate-buffered saline were added to each dilution and gently mixed. Plates were incubated for $30 \mathrm{~min}-1 \mathrm{~h}$ at room temperature. The hemagglutination inhibition titer was reported as the reciprocal of the highest dilution of serum that inhibited hemagglutination.

Statistics. Statistical differences between two groups were determined using an unpaired, two-tailed Student's $t$-test with significance set at $P<0.05$. For comparisons between $\geqslant 3$ groups, analysis was done by one-way analysis of variance followed by a Tukey's multiple comparison test with significance determined at $P<0.05$. For survival studies, a log-rank (Mantel-Cox) test was used.

SUPPLEMENTARY MATERIAL is linked to the online version of the paper at http://www.nature.com/mi

\section{ACKNOWLEDGMENTS}

This work was supported by NIH grants Al104541 (SNV and JCGB), DK048373 (AF), and Al15614 (CD), by Swiss National Science Foundation grants 320030_149511 (TR), 310030_138488, and 149511 (TC), and by Science Foundation Ireland grant 11/PI/1056 (AGB).

\section{AUTHOR CONTRIBUTIONS}

KAS and SNV carried out the study design, with advice from JCGB, AF, CAD, TR, KJT, YA, and EK-J. KAS, WL, MCP, LMP, CP, and EK-J performed experiments. FG, TR, TC, AGB, AF, CAD, KJT, YA, and EK-J provided crucial reagents and advice for this study. KAS and SNV prepared the manuscript with input and approval of all other co-authors.

\section{DISCLOSURE}

FG is an employee of Eisai. The remaining authors declared no conflict of interest.

c 2016 Society for Mucosal Immunology

\section{REFERENCES}

1. Belongia, E.A., Sundaram, M.E., McClure, D.L., Meece, J.K., Ferdinands, J. \& VanWormer, J. Waning vaccine protection against influenza A (H3N2) illness in children and older adults during a single season. Vaccine 33, 246251 (2015)

2. Flannery, B. et al. Early estimates of season influenza vaccine effectiveness-United States, January 2015. Morb. Mortal. Wkly. Rep. 64, 10-15 (2015).

3. Pebody, R.G. et al. Low effectiveness of season influenza vaccine in preventing laboratory-confirmed influenza in primary care in the United Kingdom: 2014/15 mid-season results. Eurosurveil. 20, pii 21025; doi:http://dx.doi.org/10.2807/1560-7917.ES2015.20.5.21025 (2015).

4. McLean, H.Q. et al. Influenza vaccine effectiveness in the United States during 2012-2013: variable protection by and virus type. J. Infect. Dis. 211, 1529-1540 (2015).

5. Leung, Y.H. et al. Highly pathogenic avian influenza $A$ H5N1 and pandemic $\mathrm{H} 1 \mathrm{~N} 1$ virus infections have different phenotypes in Toll-like receptor 3 knockout mice. J. Gen. Virol. 95, 1870-1879 (2014).

6. Diebold, S.S., Kaisho, T., Hemmi, H., Akira, S. \& Reis e Sousa, C. Innate antiviral responses by means of TLR7-mediated recognition of singlestranded RNA. Science 303, 1529-1531 (2004).

7. Heil, F. et al. Species-specific recognition of single-stranded RNA via tolllike receptor 7 and 8. Science 303, 1526-1529 (2004).

8. Kawai, T. \& Akira, S. The role of pattern-recognition receptors in innate immunity: update on Toll-like receptors. Nat. Immunol. 11, 373-384 (2010).

9. Seo, S.U. et al. MyD88 signaling is indispensable for primary influenza A virus infection but dispensable for secondary infection. J. Virol. 84, 12713-12722 (2010).

10. Iwasaki, A. \& Pillai, P.S. Innate immunity to influenza virus infection. Nat. Rev. Immunol. 14, 315-328 (2014).
11. Tsai, S.Y et al. DAMP molecule S100A9 acts as a molecular pattern to enhance inflammation during influenza A virus infection: role of DDX21TRIF-TLR4-MyD88 pathway. PLoS Pathog. 10, e1003848 (2014).

12. Teijaro, J.R., Walsh, K.B., Rice, S., Rosen, H. \& Oldstone, M.B. Mapping the innate signaling cascade essential for cytokine storm during influenza virus infection. Proc. Natl. Acad. Sci. USA 111, 3799-3804 (2014).

13. Pauligk, C., Nain, M., Reiling, N., Gemsa, D. \& Kaufmann, A. CD14 is required for influenza $A$ virus-induced cytokine and chemokine production. Immunobiology 209, 3-10 (2004).

14. Imai, Y. et al. Identification of oxidative stress and Toll-like receptor 4 signaling as a key pathway of acute lung injury. Cell 133, 235-249 (2008).

15. Lien, E. et al. A novel synthetic acyclic lipid A-like agonist activates cells via the lipopolysaccharide/toll-like receptor 4 signaling pathway. J. Biol. Chem. 276, 1873-1880 (2001).

16. Shirey, K.A. et al. The TLR4 antagonist Eritoran protects mice from lethal influenza infection. Nature 497, 498-502 (2013).

17. Nhu, Q.M. et al. Novel signaling interactions between proteinase-activated receptor 2 and Toll-like receptors in vitro and in vivo. Mucosal Immunol. 3, 29-39 (2010).

18. Ye, J. et al. Variations in the hemagglutinin of the $2009 \mathrm{H} 1 \mathrm{~N} 1$ pandemic virus: potential for strains with altered virulence phenotype?. PLoS Pathog. 6, e1001145 (2010).

19. Kim, H.M. et al. Crystal structure of the TLR4-MD-2 complex with bound endotoxin antagonist Eritoran. Cell 130, 906-917 (2007).

20. Roger, T. et al. Protection from lethal gram-negative bacterial sepsis by targeting Toll-like receptor 4. Proc. Natl. Acad. Sci. USA 106, 2348-2352 (2009).

21. Kawai, T., Adachi, O., Ogawa, T., Takeda, K. \& Akira, S. Unresponsiveness of MyD88-deficient mice to endotoxin. Immunity 11, 115-122 (1999).

22. Lysakova-Devine, T. et al. Viral inhibitory peptide of TLR4, a peptide derived from vaccinia protein A46, specifically inhibits TLR4 by directly targeting MyD88 adaptor-like and TRIF-related adaptor molecule. J. Immunol. 185 4261-4271 (2010).

23. Meng, G. et al. Antagonistic antibody prevents toll-like receptor 2-driven lethal shock-like syndromes. J. Clin. Invest. 113, 1473-1481 (2004).

24. Wang, $\mathrm{H}$. et al. HMG-1 as a late mediator of endotoxin lethality in mice. Science 285, 248-251 (1999).

25. Alleva, L.M., Budd, A.C. \& Clark, I.A. Systemic release of high mobility group box 1 protein during severe murine influenza. J. Immunol. 181, 1454-1459 (2008)

26. Yang, H., Antoine, D.J., Andersson, U. \& Tracey, K.J. The many faces of HMGB1: molecular structure-functional activity in inflammation, apoptosis, and chemotaxis. J. Leukoc. Biol. 93, 865-873 (2013).

27. Yang, $H$. et al. MD-2 is required for disulfide HMGB1-dependent TLR4 signaling. J. Exp. Med. 212, 5-14 (2015).

28. Tripathi, A. et al. Identification of human zonulin, a physiological modulator of tight junctions, as prehaptoglobin-2. Proc. Natl. Acad. Sci. USA 106, 16799-16804 (2009).

29. Goldblum, S.E. et al. The active Zot domain (aa 288-293) increases ZO-1 and myosin $1 \mathrm{C}$ serine/threonine phosphorylation, alters interaction between ZO-1 and its binding partners, and induces tight junction disassembly through proteinase activated receptor. FASEB J. 25, 144-158 (2011).

30. Rallabhandi, P. et al. Analysis of proteinase-activated receptor 2 and TLR4 signal transduction: a novel paradigm for receptor cooperativity. J. Biol. Chem. 283, 24314-24325 (2008).

31. Paterson, B.M., Lammers, K.M., Arrieta, M.C., Fasano, A. \& Meddling, J.B. The safety, tolerance, pharmacokinetic and pharmacodynamics effects of single doses of AT-1001 in coeliac disease subjects: a proof of concept study. Aliment. Pharmacol. Ther. 26, 757-766 (2007).

32. Rittirsch, D. et al. Zonulin as prehaptoglobin2 regulates lung permeability and activates the complement system. Am. J. Physiol. Lung Cell. Mol. Physiol. 304, L863-L872 (2013).

33. Dinarello, C.A., Simon, A. \& van der Meer, J.W. Treating inflammation by blocking interleukin-1 in a broad spectrum of diseases. Nat. Rev. Drug Discov. 11, 633-652 (2012).

34. Khazeni, N., Bravata, D.M., Holty, J.E., Uyeki, T.M., Stave, C.D. \& Gould, M.K. Systematic review: safety and efficacy of extended-duration antiviral chemoprophylaxis against pandemic and seasonal influenza. Ann. Intern. Med. 151, 464-473 (2009). 
35. Freund, B., Gravenstein, S., Elliott, M. \& Miller, I. Zanamivir: a review of clinical safety. Drug Saf. 21, 267-281 (1999).

36. Zheng, B.J. et al. Delayed antiviral plus immunomodulator treatment still reduces mortality in mice infected by high inoculum of influenza $\mathrm{A} / \mathrm{H} 5 \mathrm{~N} 1$ virus. Proc. Natl. Acad. Sci. USA 105, 8091-8096 (2008).

37. Kaneko, K., Ueda, R., Nemoto, H., lijima, H. \& Yoshimura, T. Disposition of a synthetic analog of lipid A (E5564) in rats. Xenobiotica 33, 323-339 (2003).

38. Kalil, A.C. et al. Influence of severity of illness on the effects of eritoran tetrasodium (E5564) and on other therapies for severe sepsis. Shock 36, 327-331 (2011).

39. Mullarkey, M. et al. Inhibition of endotoxin response by E5564, a novel Toll-like receptor 4-directed endotoxin antagonist. J. Pharmacol. Exp. Ther. 304, 1093-1102 (2003).

40. Piao, W. et al. A decoy peptide that disrupts TIRAP recruitment to TLRs is protective in a murine model of influenza. Cell Rep. 11, 1941-1952 (2015).

41. Means, T.K., Lien, E., Yoshimura, A., Wang, S., Golenbock, D.T. \& Fenton, M.J. The CD14 ligands lipoarabinomannan and lipopolysaccharide differ in their requirement for Toll-like receptors. J. Immunol. 163, 6748-6755 (1999).

42. Lee, H.K., Dunzendorfer, S., Soldau, K. \& Tobias, P.S. Double-stranded RNA mediated TLR3 activation is enhanced by CD14. Immunity 24, 153-163 (2006).

43. Baumann, C.L. et al. CD14 is a coreceptor of Toll-like receptors 7 and 9. J. Exp. Med. 207, 2689-2701 (2010).

44. Shirey, K.A. et al. The anti-tumor agent, 5,6-dimethylxanthenone-4-acetic acid (DMXAA), induces IFN-beta-mediated antiviral activity in vitro and in vivo. J. Leukoc. Biol. 89, 351-357 (2011).

45. Kadl, A. et al. Oxidized phospholipid-induced inflammation is mediated by Toll-like receptor 2. Free Radic. Biol. Med. 51, 1903-1909 (2011).

46. Rodrigue-Gervais, I.G. et al. Cellular inhibitor of apoptosis protein clAP2 protects against pulmonary tissue necrosis during influenza virus infection to promote host survival. Cell Host Microbe 15, 23-35 (2014).

47. Sridharan, H. \& Upton, J.W. Programmed necrosis in microbial pathogenesis. Trends Microbiol. 22, 199-207 (2014).
48. Qing, D.Y. et al. Red blood cells induce necroptosis of lung endothelial cells and increase susceptibility to lung inflammation. Am. J. Respir. Crit. Care Med. 190, 1243-1254 (2014).

49. Zhang, S. et al. Small intestinal injury in mice infected with respiratory influenza A virus: evidence for virus induced gastroenteritis. Biotechnol. Lett. 37, 1585-1592 (2015).

50. Ichinohe, T. et al. Microbiota regulates immune defense against respiratory tract influenza A virus infection. Proc. Natl. Acad. Sci. USA 108, 5354-5359 (2011).

51. Sodhi, C.P et al. Intestinal epithelial TLR-4 activation is required for development of acute lung injury after trauma/hemorrhagic shock via the release of HMGB1 from the gut. J. Immunol. 194, 4931-4939 (2015).

52. Cohen, I. et al. Differential release of chromatin-bound IL-1alpha discriminates between necrotic and apoptotic cell death by the ability to induce sterile inflammation. Proc. Natl. Acad. Sci. USA 107, 2574-2579 (2010).

53. Kim, B. et al. The interleukin-1alpha precursor is biologically active and is likely a key alarmin in the IL-1 family of cytokines. Front. Immunol. 4, 391 (2013).

54. Swain, S.L., Dutton, R.W. \& Woodlan, D.L. T cell responses to influenza virus infection: effector and memory cells. Viral Immunol. 17, 197-209 (2004).

55. Mclntire, F.C., Sievert, H.W., Barlow, G.H., Finley, R.A. \& Lee, Y. Chemical, physical, biological properties of a lipopolysaccharide from Escherichia coli K-235. Biochemistry 6, 2363-2376 (1967).

56. Teijaro, J.R. et al. Costimulation modulation uncouples protection from immunopathology in memory Tcell response to influenza virus. J. Immunol. 82, 6834-6843 (2009).

57. Blanco, J.C. et al. Receptor characterization and susceptibility of cotton rats to avian and 2009 pandemic influenza virus strains. J. Virol. 87, 2036-2045 (2013).

58. Tate, M.D., Brooks, A.G. \& Reading, P.C. The role of neutrophils in the upper and lower respiratory tract during influenza virus infection in mice. Respiratory Res. 9, 57-70 (2008).

59. Feng, C. et al. Neuraminidase reprograms lung tissue and potentiates lipolysaccharide-induced acute lung injury in mice. J. Immunol. 191, 4828-4837 (2013). 